\title{
Huesos Sesamoideos en la Mano Humana
}

\author{
Sesamoid Bones in the Human Hand
}

Olave, E.; Binvignat, O. ${ }^{* *}$; Soto, A. ${ }^{* * *} \&$ Cabezas, J. J.****

OLAVE, E.; BINVIGNAT, O.; SOTO, A. \& CABEZAS, J. J. Huesos sesamoideos en la mano humana. Int. J. Morphol., 32(1):49-53, 2014.

RESUMEN: Los huesos sesamoideos se desarrollan en el espesor de ciertos tendones o de la cápsula fibrosa que envuelve ciertas articulaciones. La prevalencia y distribución de los huesos sesamoideos en la mano es variable entre diferentes grupos poblacionales y étnicos. Basados en esta premisa realizamos un estudio para determinar su presencia en las diferentes articulaciones metacarpofalángicas (AMF) de dos grupos de individuos. Analizamos 34 radiografías de manos de adultos, chilenos, de ambos sexos, así como 19 radiografías pertenecientes a manos de niños y adolescentes. También se estudiaron 41 radiografías de manos de adultos, Brasileños, de ambos sexos, así como 16 radiografías de niños y adolescentes. En cada observación consideramos el número de huesos sesamoideos y su localización. En el grupo de individuos chilenos se observó la presencia de dos huesos sesamoideos, radial y ulnar, localizados a nivel de la AMF del pulgar en $94,1 \%$ de los casos; $44,1 \%$ tenían un hueso sesamoideo a nivel de la parte radial de la AMF del dedo indicador. A nivel de la AMF del dedo mínimo, se observó un hueso sesamoideo en 11,8\%. Del análisis de 19 radiografías de manos de niños y adolescentes, se visualizó 42\% con huesos sesamoideos a nivel de la AMF del pulgar. En el grupo de individuos brasileños, se observó que todas las manos tenían huesos sesamoideos a nivel de la AMF del pulgar; 90,2\% tenían dos huesos. En 17,1\% se encontró un hueso sesamoideo a nivel de la parte ulnar de la articulación interfalángica del pulgar. En 26,8\% se observó un hueso sesamoideo en la parte radial de la AMF del indicador y en el mismo porcentaje a nivel de la parte radial de la AMF del dedo mínimo. De las 16 radiografías de manos de niños y jóvenes brasileños, ninguna de ellas presentó tales huesos. Los datos aportados dejan en evidencia las diferencias que existen entre grupos poblacionales y étnicos.

PALABRAS CLAVE: Anatomía; Mano; Huesos sesamoideos.

\section{INTRODUCCIÓN}

Los huesos sesamoideos se desarrollan en el espesor de ciertos tendones o de la cápsula fibrosa que envuelve algunas articulaciones. Estos huesos son generalmente nódulos ovoides, de pocos milímetros de diámetro, pero varían en forma y tamaño, algunos muy grandes, como la patela. No siempre son completamente osificados y pueden ser de tejido fibroso denso, cartílago y huesos en proporciones variadas, pero la mayoría es parcialmente osificado. Ellos están generalmente incrustados en los tendones íntimamente relacionados con las caras articulares o donde los tendones se angulan agudamente alrededor de superficies óseas. En ambos locales, la cara del hueso sesamoideo relacionada con el otro hueso está cubierta por cartílago articular y desliza sobre ella. Algunos han considerado a los huesos sesamoideos como primariamente articulares, esto es incrustados en cápsulas articulares y su asociación con los tendones como secundaria. Su función no es clara, pudiendo mo- dificar la presión, disminuir la fricción y algunas veces, alterar la dirección de tracción del tendón, como lo hace la patela (Williams et al., 1995).

La prevalencia y distribución de los huesos sesamoideos en la mano es variable entre diferentes grupos poblacionales y étnicos. Generalmente estos huesos se presentan con más frecuencia en la región palmar de la $1^{\mathrm{a}}$ articulación metacarpofalángica (Amar et al., 2011), los cuales se han visto involucrados en diferentes entidades clínicas, tales como artritis, pulgar en resorte, fracturas traumáticas, entre otras (Resnick et al., 1977; Seybold \& Warnhold, 1996; Shaw et al., 2003).

Estos huesos también se presentan ocasionalmente a nivel de la articulación de la muñeca, por delante de los huesos piramidal y hamato (Tzaveas et al., 2008)

\footnotetext{
* Facultad de Medicina, Universidad de la Frontera, Temuco, Chile.

** Universidad Autónoma de Chile, Sede Talca, Chile.

**** Facultad de Medicina, Universidad Católica del Maule, Talca, Chile.
} 
Basado en lo anterior y con el propósito de determinar la presencia y su localización respecto a las diferentes articulaciones metacarpo-falángicas (AMF), estudiamos estos huesos en dos grupos de personas, uno que provenía de la región central de Chile y el otro de la región sur de Brasil.

\section{MATERIAL Y MÉTODO}

Analizamos radiografías de 34 manos, derechas e izquierdas, de individuos adultos, chilenos, de ambos sexos, y 19 de manos de niños y adolescentes. Además, se estudiaron 41 radiografías de manos de individuos adultos, brasileños, de ambos sexos, y 16 de niños y adolescentes brasileños. Consideramos el número de huesos sesamoideos, su localización, así como su posición, lateral o medial.

\section{RESULTADOS}

En el grupo de individuos chilenos se observó la presencia de dos huesos sesamoideos, radial y ulnar (Fig. 1) localizados a nivel de la AMF del pulgar en 32 muestras (94,1\%), 19 de las cuales correspondían al sexo masculino y en los dos casos restantes $(5,9 \%)$ se observó sólo un hueso sesamoideo, estos últimos pertenecientes al sexo masculino, una del lado derecho y la otra del izquierdo. A nivel de la articulación interfalángica del pulgar se observaron huesos sesamoideos en 3 casos (8,8\%), dos casos con uno y el otro con dos huesos.

Del total de las muestras estudiadas, 15 manos $(44,1 \%)$ tenían un hueso sesamoideo a nivel de la parte lateral de la AMF del dedo indicador, siendo diez de ellas del sexo masculino y cinco del femenino. A nivel de la AMF del dedo mínimo, se observó un hueso sesamoideo en 4 manos $(11,8 \%)$, dos del sexo masculino y dos del femenino.

Sólo 2 manos tenían huesos sesamoideos a nivel de la AMF del pulgar, AMF de los dedos indicador y mínimo, ambas del sexo masculino. En 14 manos (9 del sexo masculino y 5 del sexo femenino)se observó huesos sesamoideos tanto en las AMF de los dedos pulgar e indicador.

Del análisis de 19 radiografías de manos de niños, se visualizaron 8 (42\%) con huesos sesamoídeos y $11(57,9 \%)$ sin su presencia. De los que tenían estos huesos, 5 correspondía al sexo masculino, donde se observó 4 casos con dos huesos sesamoideos a nivel de la AMF del pulgar y el restante sólo con uno. De los 3 casos del sexo femenino, hubo uno con dos sesamoideos en la AMF del pulgar y los otros

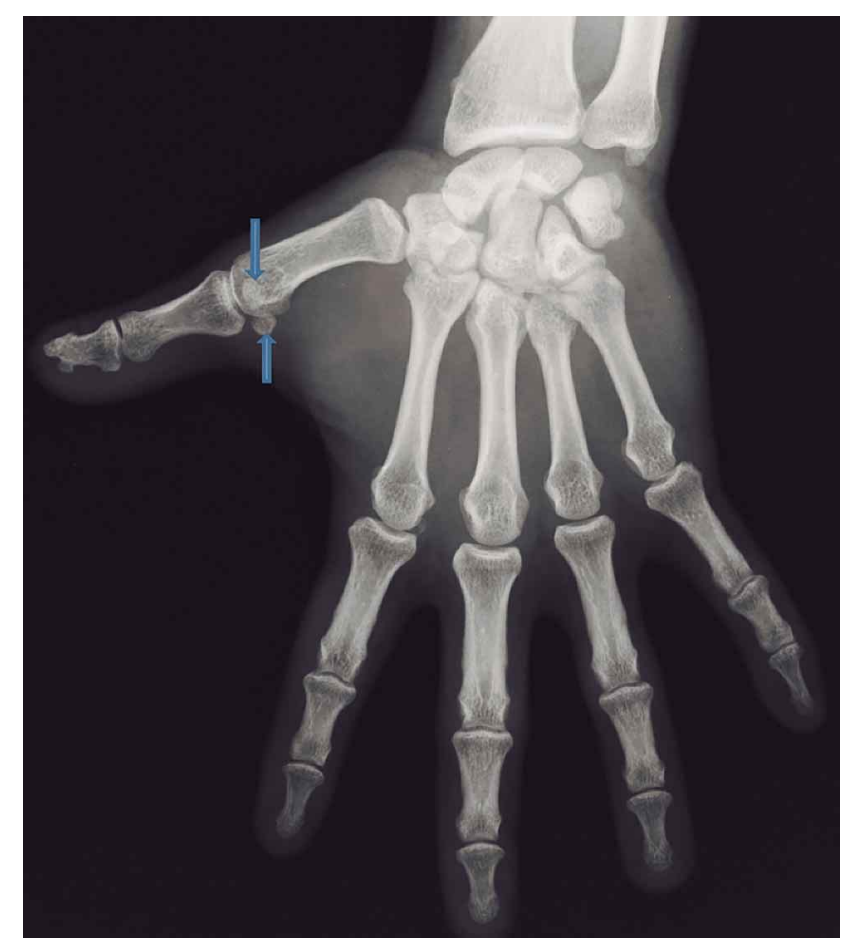

Fig. 1. Huesos sesamoideos a nivel de la articulación metecarpofalángica del pulgar (flechas).

dos con un solo hueso.

En el grupo de individuos brasileños, se observó que todas tenían huesos sesamoideos a nivel de la AMF del pulgar, 37 de ellas $(90,2 \%)$ tenían dos huesos y las restantes 4 , tenían sólo uno, de los cuales tres casos correspondieron al lado derecho.

En 7 casos $(17,1 \%)$ se encontró un hueso sesamoideo a nivel de la parte ulnar de la articulación interfalángica del pulgar, de los cuales sólo uno correspondía al lado izquierdo.

En 11 casos $(26,8 \%)$ se observó un hueso sesamoideo en la parte radial de la AMF del dedo indicador (Fig. 2), de los cuales, 4 correspondían al lado izquierdo. En otros 11 casos $(26,8 \%)$ se visualizó un hueso sesamoideo a nivel de la AMF del dedo mínimo (Fig. 3).

La presencia de varios huesos sesamoideos en una misma mano se presentó de la siguiente forma: sesamoideos en las AMF de los dedos pulgar, indicador y mínimo se observó en 5 manos; en las AMF del pulgar y en la AMF del dedo mínimo se presentó en 7 manos y su presencia en la AMF del pulgar e indicador se observó en 5 manos; también en la AMF del pulgar como a nivel de la articulación interfalángica de este dedo en 7 manos. La presencia de huesos sesamoideos sólo a nivel de la AMF del pulgar la tuvieron 17 manos. 
De las 16 radiografías de manos de niños y jóvenes brasileños, ninguna de ellas presentó tales huesos.

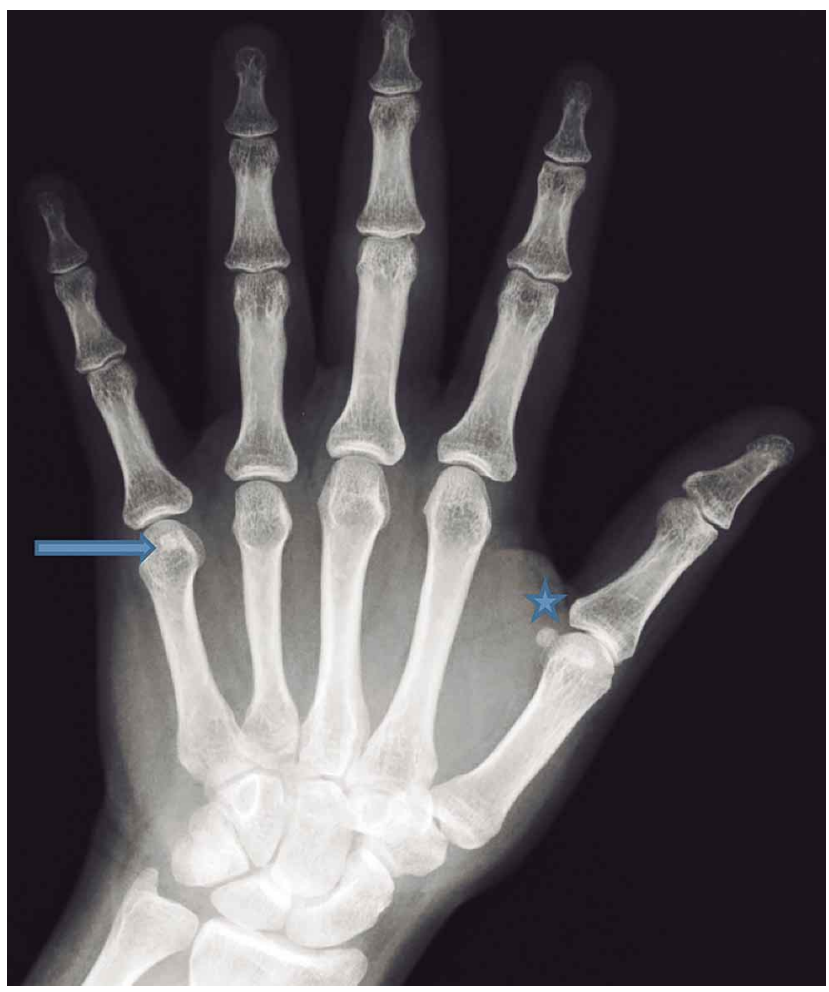

Fig. 2. Hueso sesamoideo a nivel de la articulación metacarpofalángica del dedo mínimo (flecha) y en la del pulgar (estrella).

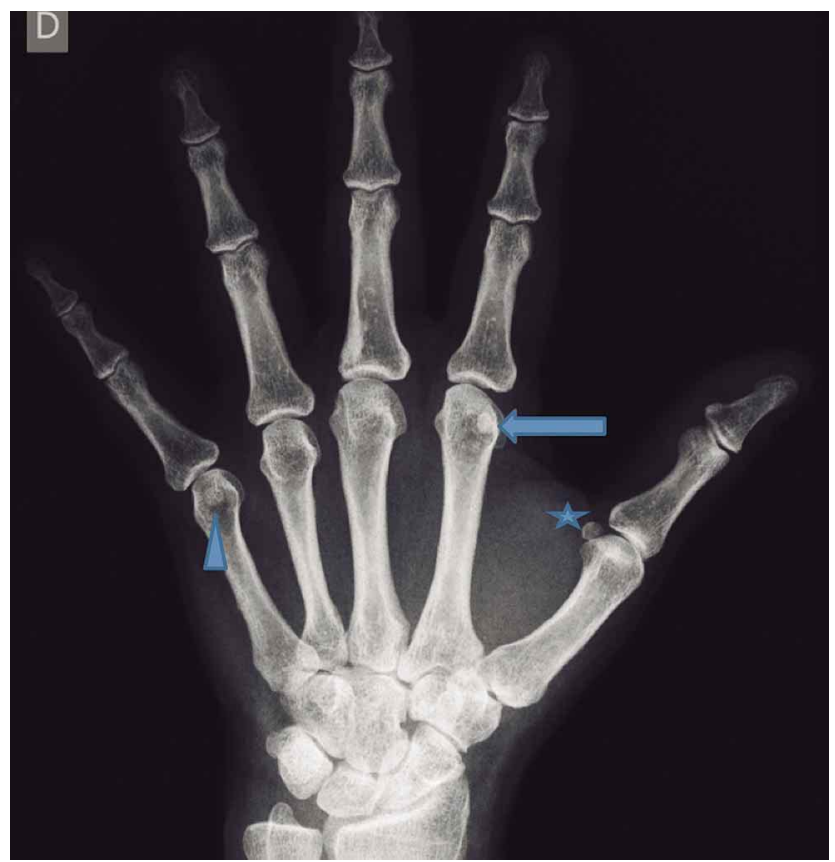

Fig. 3. Hueso sesamoideo a nivel de la articulación metacarpofalángica del dedo indicador (flecha), en la del pulgar (estrella) y en la del dedo mínimo (punta de flecha).

\section{DISCUSIÓN}

La denominación de hueso sesamoideo deriva del griego sesamen (= sésamo) y de oides (= forma de), debido a la semejanza de estos pequeños huesos y las semillas de sésamo (Medeiros Fernandes, 1999), aunque el término de hueso sesamoideo es atribuído a Galeno, quien describió pequeños huesos en las manos y pies. Sin embargo, la comparación con estas semillas está muy poco justificada, porque los huesesillos en cuestión tienen muy remota semejanza con ellas (Testut \& Latarjet, 1972).

Estos huesos han sido clasificados en periarticulares e intratendinosos, donde en la mano, los primeros están exclusivamente en la cara palmar, por lo tanto en el lado de la flexión (Testut \& Latarjet). De acuerdo a la literatura estos huesos son variables entre diferentes grupos poblacionales o étnicos. Testut \& Latarjet han señalado que existen de 2 a 7 por mano, dos son constantes, los que corresponden a la AMF del pulgar, los otros son inconstantes y sólo aparecen en algunos individuos.

Fawcett (1896) analizó 38 manos y observó que a nivel de la AMF del pulgar hay dos huesos sesamoídeos, uno radial y otro ulnar; que a nivel de la articulación interfalángica del pulgar había sólo uno en la parte palmar del ligamento capsular en 68,5\%; que a nivel de la AMF del indicador había un sesamoideo radial en 55,2\% y a nivel de la AMF del dedo mínimo se presentó en 78\%. Tales valores son bastante más altos que los obtenidos en nuestra serie.

Por su parte, Joseph (1951) analizó a 309 individuos europeos (174 hombres y 135 mujeres), 31 personas de origen indio y 30 procedentes de Africa, encontrando dos huesos sesamoideos en la AMF del pulgar en el $100 \%$ de los casos; encontró uno a nivel de la AMF del dedo indicador en $70 \%$ y a nivel de la AMF del dedo mínimo en $35 \%$, valores más altos que los de nuestra serie, excepto los dos localizados a nivel de la AMF del pulgar, cuya presencia es concordante con lo descrito por los autores mencionados.

Este autor también indicó que los huesos sesamoídeos interfalángicos nunca aparecen antes que los metacarpofalángicos en el pulgar, concluyendo que estos últimos aparecen entre los 12 y 15 años en hombres y entre 10 y 12 años en mujeres. De acuerdo a este autor, individuos jóvenes pueden no haber desarrollado los huesos sesamoídeos, lo que podría ser una razón para explicar algunas diferencias de porcentaje, admitiendo también que el plano en que se obtuvo la radiografía podría también influir en tales diferencias. 
Dharap et al. (2007) analizaron 400 radiografías de manos de una población árabe procedente de Bahrain y observaron que todas las radiografías de manos adultas (213) presentaron dos huesos sesamoideos palmares a la AMF del pulgar, resultado con el cual concordamos ya que en nuestra muestra se observó un valor cercano a 100\%; un hueso sesamoideo fue encontrado palmar a la AMF del dedo indicador en 86 manos (49 derechas y 37 izquierdas), lo que corresponde a un $40,5 \%$, valor semejante al encontrado en el presente estudio en el grupo de individuos chilenos $(44,1 \%)$, sin embargo en el grupo de individuos brasileños lo observamos sólo en un 26,8\%. Frecuencias menores han sido reportadas en individuos de Malawi, Africa Central, por Msamati \& Igbigbi (2001) quienes observaron en este grupo, sólo un 4,8\% de presencia de sesamoideos a nivel de la articulación mencionada.

A nivel del dedo mínimo, estos autores encontraron solo un hueso sesamoideo, palmar a la AMF en 96 manos (54 derechas y 42 izquierdas), mostrando una incidencia de $45,3 \%$, valor mucho más alto que el encontrado en nuestra serie de individuos provenientes del Sur de Brasil y en el que sólo apreciamos estos huesos en el 26,8\%, al nivel indicado por estos autores y más aún, del grupo de individuos chilenos en que sólo se presentaron un 11,8\% de los casos.

Huesos sesamoideos palmares a las AMF de los dedos medio y anular fueron raros, observando solo un 2,3\% y $1,5 \%$, respectivamente, presencia no observada en las series analizadas en este estudio.

Estos mismos autores indicaron que 61 manos $(28,6 \%)$ presentaron un hueso sesamoideo palmar a la AMF del pulgar, diferente del valor aportado por Joseph que señaló un $74 \%$ de presencia a este nivel, no encontrando otros huesos a nivel de las articulaciones de los demás dedos. Para esta presencia, nuestra serie mostró solo un $17,1 \%$ en el grupo brasileño y un $8,8 \%$ en el grupo chileno, y coincidiendo con estos autores, no se observaron huesos sesamoideos en las otras articulaciones interfalángicas.

Por su parte, Amar et al. estudiaron una población mediterránea a través de 442 radiografías de pacientes adultos y encontraron una incidencia de 99,5\% (440) a nivel de la AMF del pulgar, $42,3 \%$ a nivel de la segunda (187) y $41,1 \%$ (182) a nivel de la $5^{\text {a }}$ AMF. También informaron de la presencia de huesos sesamoideos a nivel de la $1^{\mathrm{a}}$ articulación interfalángica en 26,2\% (116). Como se puede apreciar estos resultados son coincidentes con los de Dharap et al., con valores de presencia más altos, que los nuestros, en ambos grupos presentados, excepto a nivel de la segunda articulación en el grupo de individuos chilenos, donde se observó un $44,1 \%$.
Recientemente, Kose et al. (2012) señalaron que los huesos sesamoideos de la mano se encuentran en la superficie de la palma de varias articulaciones. Hay dos constantes en la AMF del pulgar, de los cuales el medial es el más grande; uno en la articulación interfalángica del pulgar; uno es con frecuencia presente en la articulación correspondiente del dedo meñique, y uno (o dos) en la articulación del dedo indicador. Los huesos sesamoideos también se encuentran ocasionalmente en las AMF de los dedos medio y anular y en la articulación interfalángica distal del dedo indicador. Estos autores analizaron 922 Radiografías de un Grupo de Pacientes de Nacionalidad turca y encontraron que a nivel de la AMF del pulgar, dos huesos sesamoideos estaban presentes en todos los sujetos. Un hueso sesamoideo fue visto palmar a la articulación interfalángica del dedo pulgar en 197 manos (21,3\%). Un hueso sesamoideo se encontró palmar a la AMF del dedo indicador en 338 manos (36,6\%). A nivel del dedo meñique un hueso sesamoideo fue observado palmar a la AMF en 491 manos $(53,2 \%)$. Los huesos sesamoideos palmares a las AMF de los dedos medio y anular eran poco frecuentes, la incidencia a este nivel fue de $1,3 \%$ (12 manos) y 0,9\% (8 manos), respectivamente. Nuestros resultados se aproximan un poco más a los de estos últimos autores, sobretodo en relación al hueso sesamoideo observado a nivel de la AMF del pulgar y a la AMF del indicador, cuyos valores en los individuos brasileños fueron $17,1 \%$ y $26,8 \%$, respectivamente. Para la presencia a nivel de la AMF del indicador, el registro obtenido en el grupo de individuos chilenos $(44,1 \%)$ es semejante al resultado de estos últimos autores. Resalta el valor a nivel de la AMF del dedo mínimo, donde nuestro resultado es muy inferior al del estudio precedente. Sin embargo, coincidimos plenamente con los valores señalados para la existencia de dos huesos sesamoideos a nivel de la AMF del pulgar.

Las fracturas de los huesos sesamoídeos son una lesión rara y es un indicador de la magnitud de las lesiones por hiperextensión (Deabate et al., 2011). Han sido descritas en el sesamoídeo radial y ulnar de la AMF del pulgar (Bianchi et al., 1993; Altun et al., 1998; Shaw et al.; Deabate et al.).

Los datos aportados dejan en evidencia las diferencias que existen entre grupos poblacionales y étnicos, principalmente en lo que se refiere a los huesos sesamoideos de las AMF de los dedos indicador y mínimo, así como los de la articulación interfalángica del pulgar. Por otra parte, es importante considerar la edad de los individuos, ya que su presencia en niños y jóvenes es reducida o pueden no estar presentes. 


\section{OLAVE, E.; BINVIGNAT, O.; SOTO, A. \& CABEZAS, J. J.Sesamoid bones in the human hand. Int. J. Morphol., 32(1):49-53, 2014.}

SUMMARY: Sesamoid bones develop in certain tendons or thickness of the fibrous capsule which surrounds certain joints. The prevalence and distribution of sesamoid bones in the hand is variable among different populations and ethnic groups. Based on this premise we conducted a study to determine its presence in different metacarpophalangeal joints (MPJ) of two groups of individuals. We analyzed 34 radiographs of adult hands, Chileans, of both sexes, and 19 radiographs belonging to the hands of children and adolescents. Radiographs of 41 hands of adult Brazilians people, of both sexes, as well as radiographs of 16 children and adolescents were also studied. In each observation we consider the number of sesamoid bones and their location. In the group of Chilean individuals, we observed the presence of two sesamoid bones, located at the level of MPJ thumb in $94.1 \%$ of cases; $44.1 \%$ had one sesamoid bone in the radial side of MPJ of the index finger. At the level of the MPJ of the little finger, one sesamoid bone was observed in $11.8 \%$. An analysis of 19 radiographs of the hands of children and adolescents, in $42 \%$ of the cases we found sesamoid bones at MPJ of the thumb. In the group of Brazilian individuals, we observed that all hands had sesamoid bones at the level of MPJ of the thumb; $90.2 \%$ had two bones. In $17.1 \%$ one sesamoid bone at the ulnar side of the interphalangeal joint of the thumb was found. In $26.8 \%$ one sesamoid bone in the radial part of the MPJ of the index finger and the same percentage in the radial side of the MPJ of the little finger was observed. We not observed such bones in the radiographs of the hands of children and young Brazilians. The data provided are evidence of differences between populations and ethnic groups.

\section{KEY WORDS: Anatomy; Hand; Sesamoid bones.}

\section{REFERENCIAS BIBLIOGRAFICAS}

Altun, M.; Erdil, H.; Sahin, H. \& Gumusburun, E. Fracture of the ulnar sesamoid of the metacarpophalangeal joint of the thumb: a case report. Clin. Anat., 11(1):60-1, 1998.

Amar, E.; Rozenblat, Y. \& Chechik, O. Sesamoid and accessory bones of the hand - An epidemiologic survey in a Mediterranean population. Clin. Anat., 24(2):1837, 2011.

Bianchi, S.; Abdelwahab, I. F.; Zwass, A. \& Molini, L. Fractures of the sesamoid bone of the thumb. Bull. Hosp. Jt. Dis., 53(3):7-10, 1993.

Deabate, L.; Garavaglia, G.; Lucchina, S. \& Fusetti, C. Fracture of the radial sesamoid bone of the thumb: an unusual fracture. Chin. J. Traumatol., 14(5):309-11, 2011.

Dharap, A. S.; Al-Hashimi, H.; Kassab, S. \& Abu-Hijleh, M. F. Incidence and ossification of sesamoid bones in the hands and feet: A radiographic study in an Arab population. Clin. Anat., 20(4):416-23, 2007.

Fawcett, E. On the sesamoid bones of the hand: A skiagraphic confirmation of the work done by Pfitzner. J. Anat. Physiol., 31(Pt. 1):157-61, 1896.

Joseph, J. The sesamoid bones of the hand and the time of fusion of the epiphyses of the thumb. J. Anat., 85(3):230-41, 1951.

Kose, O.; Guler, F.; Turan, A.; Ecanbora, K. \& Akalin, S. Prevalence and distribution of sesamoid bones of the hand. A radiographic study in Turkish subjects. Int. J. Morphol., 30(3):1094-9, 2012.

Msamati, B. C. \& Igbigbi, P. S. Radiographic appearance of sesamoid bones in the hands and feet of Malawian subjects. Clin. Anat., 14(4):248-53, 2001.
Medeiros Fernandes, G. Eponimia: glossário de termos eponimos em Anatomia; Etimologia: dicionário etimológico da nomenclatura anôtomica. São Paulo, Pleiade Ltda., 1999.

Resnick, D.; Niwayama, G. \& Feingold, M. The sesamoid bones of the hands and feet: participators in arthritis. Radiology, 123(1):57-62, 1977.

Seybold, E. A. \& Warnhold, L. G. Impingement of the flexor pollicis longus tendon by an enlarged radial sesamoid causing trigger thumb: a case report. J. Hand Surg. Am., 21(4):619-20, 1996.

Shaw, M.; Lyburn, I. D.; Torreggiani, W. C. \& Watura, R. Conminuted fracture of the ulnar sesamoid of the metacarpophalangeal joint of the thumb: an uncommon injury. J. Emerg.Med., 24(4):437-9, 2003.

Testut, L. \& Latarjet, A. Tratado de Anatomía Humana. $9^{a}$ ed. Barcelona, Salvat, 1972. v.3, p.1142.

Tzaveas, A.; Paraskevas, G.; Gekas, Ch.; Vretakkos, A.; Antoniou, K. \& Spyridakis, I. Anatomical variations of co-existence of $4^{\text {th }}$ and $5^{\text {th }}$ short metacarpal bones, sesamoid ossicles and exostoses of ulna and radius in the same hand: a case report. Cases J., 1(1):281, 2008.

Williams, P. L.; Warwick, R.; Dyson, M. \& Bannister, L. H. Gray Anatomía. 37ª ed. Rio de Janeiro, Guanabara-Koogan ,1995. v.2.

Dirección para Correspondencia:

Prof. Dr. Enrique Olave

Facultad de Medicina

Universidad de La Frontera

Casilla $54 \mathrm{D}$

Temuco - CHILE

Recibido : 16-10-2013

Email: enrique.olave@ufrontera.cl 